\title{
Performance of Model-Based vs. Permutation Tests in the HEALing (Helping to End Addiction Long- TermSM) Communities Study, a Covariate Constrained Cluster Randomized Trial
}

\section{Xiaoyu Tang ( $\square$ rainie@bu.edu )}

Boston University https://orcid.org/0000-0002-7236-3201

Timothy Heeren

Boston University School of Public Health

Philip M Westgate

University of Kentucky College of Public Health

Daniel J. Feaster

University of Miami

\section{Soledad A. Fernandez}

The Ohio State University College of Medicine

\section{Nathan Vandergrift}

RTI International

Debbie M. Cheng

Boston University School of Public Health

\section{Research Article}

Keywords: Covariate constrained randomization, Model-based tests, Permutation tests, Cluster randomized trials, Negative binomial regression

Posted Date: February 7th, 2022

DOI: https://doi.org/10.21203/rs.3.rs-1256557/v1

License: (c) (i) This work is licensed under a Creative Commons Attribution 4.0 International License.

Read Full License 
1 Performance of Model-based vs. Permutation Tests in the HEALing (Helping

2 to End Addiction Long-term ${ }^{\mathrm{SM}}$ ) Communities Study, a Covariate Constrained Cluster Randomized Trial

\author{
Xiaoyu Tang1, Timothy Heeren', Philip M Westgate ${ }^{2}$, Daniel J. Feaster ${ }^{3}$, Soledad A. \\ Fernandez $^{4}$, Nathan Vandergrift ${ }^{5}$, Debbie M. Cheng ${ }^{1}$ \\ ${ }^{1}$ Department of Biostatistics, Boston University School of Public Health, Boston, USA \\ ${ }^{2}$ Department of Biostatistics, University of Kentucky College of Public Health, Lexington, USA \\ ${ }^{3}$ Department of Public Health Sciences, University of Miami, Florida, USA and Columbia \\ University School of Social Work, New York, USA \\ ${ }^{4}$ Department of Biomedical Informatics, The Ohio State University College of Medicine, \\ Columbus, USA \\ ${ }^{5}$ RTI International, North Carolina, USA \\ Correspondence \\ Xiaoyu Tang, Department of Biostatistics, Boston University School of Public Health, 801 \\ Massachusetts Avenue, Boston, MA 02219, USA. \\ Email: rainie@bu.edu
}

Abstract

Background: The HEALing (Helping to End Addiction Long-term ${ }^{\mathrm{SM}}$ ) Communities Study (HCS) is a multi-site parallel group cluster randomized wait-list comparison trial designed to evaluate the effect of the Communities That Heal (CTH) intervention compared to usual care on opioid overdose deaths. Covariate constrained randomization (CCR) was applied to balance the community-level baseline covariates in the HCS. The purpose of this paper is to evaluate the

33 simulation study to evaluate Type I error rates and power for model-based and permutation tests

34 for the multi-site HCS as well as for a subgroup analysis of a single state (Massachusetts). We

35 also investigated whether the maximum degree of imbalance in the CCR design has an impact on the performance of the tests. 
38 Methods: The primary outcome, number of opioid overdose deaths, is count data assessed at the

39 community-level that will be analyzed using a negative binomial regression model. We

40 conducted a simulation study to evaluate the Type I error rates and power for 3 tests: 1) Wald-

41 type t-test with small-sample corrected empirical standard error estimates; 2) Wald-type z-test

42 with model-based standard error estimates; and 3) permutation test with test statistics calculated

43 by the difference in average residuals for the two groups.

45 Results: Our simulation results demonstrated that Wald-type t-tests with small-sample corrected

46 empirical standard error estimates from the negative binomial regression model maintained

47 proper Type I error. Wald-type z-tests with model-based standard error estimates were anti-

48 conservative. Permutation tests preserved Type I error rates if the constrained space was not too

49 small. For all tests, power was high to detect the hypothesized $40 \%$ reduction in opioid overdose

50 deaths for the intervention vs. comparison group both for the overall HCS and the subgroup

51 analysis of Massachusetts (MA).

53 Conclusions: Based on the results of our simulation study, the Wald-type t-test with small-

54 sample corrected empirical standard error estimates from a negative binomial regression model is

55 a valid and appropriate approach for analyzing cluster-level count data from the HEALing

56 Communities Study.

57

58 Trial registration: ClinicalTrials.gov http://www.clinicaltrials.gov; Identifier: NCT04111939 
60 Keywords: Covariate constrained randomization, Model-based tests, Permutation tests, Cluster

61 randomized trials, Negative binomial regression

64 1. Background

65 Cluster randomized trials are widely used when researchers are aiming to study an intervention 66 delivered at a group level (e.g. community, hospital, or school). However, techniques such as

67 stratified randomization may not provide sufficient balance of key baseline characteristics given

68 randomization at the cluster level [1]. Balance of baseline covariates is important not only to

69 improve power and precision[2], but also for the validity and credibility of studies as imbalance

70 of key covariates may bias results[3]. Many techniques exist to address the problem of covariate

71 imbalance in cluster randomized trials, such as matching[4], stratification[5] and

72 minimization[6]. Moulton proposed the covariate-constrained randomization (CCR) method

73 which can balance multiple covariates simultaneously without the risk of over-stratification [7].

74 With CCR, all clusters are recruited prior to randomization and a set of key confounding

75 variables is pre-specified along with the maximum degree of acceptable imbalance between

76 randomized arms for each confounder. Among the set of group allocations that satisfy the pre-

77 specified criteria for imbalance, i.e. the constrained randomization space, one is randomly

78 selected to generate all randomized assignments for the trial.

80 Some cluster-randomized trials may not have a sufficient number of clusters to ensure that large

81 sample model-based inference is appropriate [8]. The permutation test is a method that does not

82 require distributional assumptions and can be performed based on different test statistics [9]. 
83 Hence, it may be more appropriate than the model-based test in some settings. Fu et al.

84 compared the performance of model-based and permutation tests from a generalized linear mixed

85 model in cluster-randomized trials that do not use CCR and found that the permutation test has

86 the advantage of preserving nominal Type I error for small studies and large inter-cluster

87 correlation[10]. In terms of power, both methods had similar results. Murray demonstrated that if

88 cluster randomization provides balance on confounders at baseline, Type I error rate and power

89 are similar for permutation tests and mixed-model regression[11].

90

91 Currently there is limited research comparing permutation tests vs. model-based tests in the

92 setting of cluster randomized trials that use CCR. Li et al. (2016) compared adjusted permutation

93 tests and adjusted linear mixed effect model-based tests in the setting of CCR, and concluded

94 that in analyses adjusting for group-level covariates, both permutation tests and model-based

95 tests can maintain the correct Type I error rate as long as the number of possible allocations in

96 the constrained randomization space is not too small [12]. Li et al. (2017) compared permutation

97 tests and model-based tests for randomized trials with binary outcomes in the setting of CCR.

98 They found that when the prognostic group level covariates are balanced by CCR at the design

99 stage, both adjusted model-based tests (linearization F-test, KC-corrected GEE t-test) and

100 adjusted permutation tests gain power and preserve test size compared to unadjusted analyses

101 [13]. In both of the studies by $\mathrm{Li}$, randomization is at the cluster-level while the unit of analysis

102 is at individual level.

103

104 The purpose of this paper is to evaluate the performance of model-based tests and permutation 105 tests in the covariate constrained cluster-randomized HEALing (Helping to End Addiction Long- 
106 term $^{\mathrm{SM}}$ ) Communities Study (HCS). The HCS is a multi-site parallel group cluster randomized 107 wait-list comparison trial of the Communities That Heal (CTH) intervention[14-16]. The HCS

108 was designed to evaluate the impact of the CTH intervention compared to usual care on opioid

109 overdose deaths among 67 communities. The primary hypothesis is that communities in the

110 intervention group will have a reduction in opioid-related deaths compared to those in the

111 comparison group. This study was conducted in 4 states: Massachusetts, Kentucky, New York,

112 and Ohio. CCR was used to balance 3 community-level baseline covariates: 1) rural/urban status,

113 2) population size 3) baseline opioid death rate. In the HCS, all key variables (i.e. outcomes and

114 covariates) are aggregate community-level data. The primary outcome, number of opioid

115 overdose deaths, is count data assessed at the community-level and analyzed using a negative

116 binomial regression model.

118 As previous research on CCR focused on settings with individual-level data and did not analyze 119 group-level count outcomes, it is unclear whether the results hold in the HCS setting. To address

120 this question, we conducted a series of simulation studies to evaluate the performance of model-

121 based tests and permutation tests in terms of Type I error and power for the HCS setting. Our

122 primary goal is to evaluate test performance for the HCS based on the pre-specified CCR

123 constraints. The secondary objective is to investigate whether the number of clusters impacts test

124 performance by assessing test performance for both the overall 4-site study as well as for a single

125 state (i.e. Massachusetts only). We also explore the impact of maximum degree of covariate

126 imbalance by assessing results using alternative constraints for the CCR.

127 In section 2, we present the specific model-based and permutation tests we examined and

128 describe our simulation methods. In section 3, we present the results of the simulation study, 
129 summarizing the performance of the model-based tests and permutation tests in a range of

130 scenarios. In section 4, we discuss the results and limitations of our study.

132 2. Methods

$133 \quad 2.1$ Setting

134 The HCS was designed to evaluate the impact of the CTH intervention on opioid overdose 135 deaths in 67 highly affected communities in Massachusetts, Kentucky, New York and Ohio.

136 Massachusetts, Kentucky and New York each enrolled 16 communities and Ohio recruited 19

137 communities. The 67 communities were randomly assigned to either the intervention group or a

138 wait-list comparison group using CCR stratified by state. CCR was used to ensure balance

139 between intervention and comparison communities on three covariates at baseline. Specifically,

140 the criteria were 1) less than a 0.2 standard deviation difference in the mean baseline opioid

141 overdose death rate between intervention and comparison communities; 2) less than a 0.2

142 standard deviation difference in the overall mean population size between intervention and

143 comparison communities and 3) an equal number of rural and urban communities when there are

144 an even number of communities and a difference of 1 otherwise. Based on these pre-specified

145 constraints, the following were the number of acceptable allocations (total possible allocations)-

146 Massachusetts: 644 (12,870); Kentucky: 216 (12,870); New York: 650 (12,870); Ohio: 6,602

147 (92,378). The primary objective of the simulation study is to verify that the planned model-based

148 test will maintain the proper Type I error rate for the overall HCS and compare its performance

149 to permutation tests that directly account for the CCR. Secondarily we explore the performance

150 of the model-based and permutation tests for a single site (i.e. Massachusetts) to assess whether a

151 smaller number of clusters impacts test performance. In addition, we investigate the impact of 
152 maximum degree of covariate imbalance on the performances of the tests for both the overall

153 HCS and the single site analysis of Massachusetts.

155 2.2 Planned HCS Analysis: Negative Binomial Regression with Small-Sample Correction

156 Data for the HCS study will be measured at the community level, and the planned primary

157 analysis for the HCS study will use negative binomial regression as the dependent variable $Y$,

158 number of opioid overdose deaths, is count data. Negative binomial regression is a generalization

159 of Poisson regression that does not require the assumption that the variance of the outcome count

160 is equal to the mean. For the HCS, we expect over-dispersion in the data, i.e. $E(Y)<\operatorname{Var}(Y)$.

161 As described by Westgate et al., the proposed negative binomial model will utilize small-sample

162 adjusted empirical standard error estimates and degrees of freedom of the t-statistic equal to the

163 number of communities minus the number of regression parameters[16]. The negative binomial

164 regression model that will be used to analyze the number of opioid overdose deaths is:

165

$$
\begin{aligned}
\mu_{i}=E\left(Y_{i}\right)= & \exp \left(\log \left(n_{i}\right)+\beta_{0}+\beta_{\text {int }} X_{i_{\text {intervention }}}+\beta_{\text {urban }} X_{i_{\text {urban }}}+\beta_{d r} X_{i_{\text {deathrate }}}\right. \\
& \left.+\beta_{N Y} X_{i_{N Y}}+\beta_{K Y} X_{i_{K Y}}+\beta_{M A} X_{i_{M A}}\right)
\end{aligned}
$$

167 where $i$ indexes the individual community, baseline population $n_{i}$ is the offset variable, $\beta_{0}$ is 168 intercept and $\beta_{\text {int }}, \beta_{\text {urban }}, \beta_{d r}, \beta_{N Y}, \beta_{K Y}, \beta_{M A}$ are corresponding regression coefficients for the 169 intervention $\left(X_{i_{\text {intervention }}}\right)$, rural/urban status $\left(X_{i_{\text {urban }}}\right)$, baseline opioid death rate $\left(X_{i_{\text {deathrate }}}\right)$, 170 and state indicators for $\mathrm{NY}\left(X_{i_{N Y}}\right), \mathrm{KY}\left(X_{i_{K Y}}\right)$ and $\mathrm{MA}\left(X_{i_{M A}}\right)$, respectively. Baseline population, $171 \mathrm{rural} / \mathrm{urban}$ status, opioid death rate and states are included in the model to improve efficiency as 172 they were the covariates used in the CCR. The dispersion parameter k satisfies $\operatorname{var}\left(Y_{i} \mid X_{i}\right)=$ 
$173 \mu_{i}+k \mu_{i}^{2}$. The small-sample corrected empirical standard error estimate that will be used in the

174 primary HCS analyses follows the suggestion from Ford and Westgate, which is the average of

175 the small-sample corrected empirical estimators proposed by Mancl and DeRouen, and

176 Kauermann and Carroll[17-20].

177

178

2.3 Tests Evaluated in the Simulation Study

179

2.3.1 Model-based Tests

180 We evaluated 2 model-based tests in our simulation study:

181 1) The first model-based test is from the planned primary analysis of the HCS described above, i.e. the Wald-type t-statistic utilizing small-sample corrected empirical standard errors $\left(S E_{c}\right)$ from the negative binomial regression model: $\frac{\widehat{\beta}_{\text {int }}}{S E_{c \beta_{\text {int }}}}$. We assume that the tstatistic under null hypothesis follows a t distribution with degrees of freedom equal to $n-p-1$, where $\mathrm{n}$ is the number of communities and $\mathrm{p}$ is the number of covariates. For HCS where $n=67$ and $p=6$, the number of degrees of freedom is therefore 60 .

2) The second model-based test is the Wald-type z-statistic from a standard negative binomial regression using model-based standard errors(SE): i.e, $\frac{\widehat{\beta}_{\text {int }}}{S E_{\beta_{\text {int }}}}$.

189 We note that neither of the above model-based tests directly accounts for the constrained 190 randomization space.

\section{$192 \quad$ 2.3.2 Permutation Test}

193 A permutation test is a statistical test in which the distribution of the test statistics under null

194 hypothesis is obtained by calculating all possible values of the test statistic under all possible 
rearrangements of the study groups[21]. Permutation tests do not make assumptions about the

196 sampling distribution of test statistics. We focused on the difference in the average residuals as

197 the test statistic for our permutation test, an approach proposed by Gail et. al [22]. The residuals,

$198 r_{i}$, were defined as as $r_{i}=Y_{i}-\hat{Y}_{i}$. To calculate the fitted value, $\hat{Y}_{i}$, for community $i$, we fitted the

199 negative binomial regression on rural/urban, baseline opioid overdose death rate for community $i$

200 and $\log$ of population size for community $i$ as offset (note that intervention is not included in this

201 model). We then obtained the average residual for the intervention group $\overline{r_{1}}=n_{1}^{-1} \sum_{j=1}^{n_{1}} r_{j}$ where

$202 n_{1}$ is the sample size in the intervention group, and the average residual for the comparison

203 group, i.e. $\overline{r_{0}}=n_{0}^{-1} \sum_{j=1}^{n_{0}} r_{j}$ where $n_{0}$ is the sample size in the comparison group. Under the null

204 hypothesis of no intervention effect, these residuals are independent of the intervention

205 assignments. The resulting difference in average residuals test statistic is defined as $\mathrm{U}=\overline{r_{1}}-\overline{r_{0}}$.

206 We calculated the average residual test statistics for each permutation of the treatment

207 assignment in the permutation space to obtain the sampling distribution for the statistics. The

208 space of the permutation test was determined based on the constrained randomization space of

209 the HCS.

2112.4 Simulation Study

212 We conducted a series of simulations to evaluate both Type I error and power for the model-

213 based and permutation tests. For Type I error, we assumed that the true intervention effect is 0

214 and used a 2-sided p-value when evaluating Type I error. For power, consistent with the design

215 of the HCS, we assumed that the intervention group would experience a $40 \%$ reduction in opioid

216 overdose deaths in the $2^{\text {nd }}$ year of implementation while the wait-list comparison group would

217 experience no change. In addition to this scenario, we also assessed power for smaller effect 
218 sizes of $20 \%$ and $30 \%$. For power, we report the probability of rejecting the null in the

219 hypothesized direction when testing at the two-tailed 0.05 level. We ran 5000 iterations for the

220 simulation. We note that with 5000 iterations, the precision of the simulation study, in terms of

221 the resulting width of the $95 \%$ CI for the Type I error (alpha=0.05), is $\pm 1.96 \times \sqrt{\frac{0.05(1-0.05)}{5000}}=$

$222 \pm 0.006$. For each iteration, we obtained a p-value corresponding to each model-based test and

223 permutation test. We compared these p-values to the nominal level of 0.05 to determine the Type

224 I error and power for each test. The Type I error and power were calculated conditionally on the 225 particular randomized group allocation that was observed for the HCS.

227 2.4.1 Maximum Degree of Covariate Imbalance in the CCR Design

228 We investigated whether the maximum degree of covariate imbalance in the CCR design

229 influenced the performance of tests by examining tighter constraints than those applied in the

230 HCS, both for the overall HCS and within the single-site analysis of Massachusetts. We focused

231 only on the constraints for continuous covariates, i.e. population size and opioid death rate, as the

232 constraint for urban/rural status (i.e. equal for even numbers and differ by no more than 1 for odd

233 numbers of communities) could not be further tightened. For population size and opioid death

234 rate, we considered the following range of constraints: a difference between the 2 arms $<0.2$ SD,

$235 \quad 0.1 \mathrm{SD}$ and $0.05 \mathrm{SD}$ of the overall mean. The allowable degree of covariate imbalance is directly

236 related to the size of the constrained randomization space (i.e. number of acceptable allocations).

237 The constraints we evaluated correspond to the following number of acceptable allocations for

238 the overall HCS: $5.74 \times 10^{11}(0.2 \mathrm{SD}), 6.23 \times 10^{7}(0.1 \mathrm{SD})$ and $921,984(0.05 \mathrm{SD})$, out of

$2391.96 \times 10^{17}$ allocations in the unconstrained sampling space. We note that for the constraint with

$240 \quad 0.05 \mathrm{SD}$, there is no acceptable allocation for Kentucky. Hence, we evaluated only the 
241 constraints with $0.2 \mathrm{SD}$ and $0.1 \mathrm{SD}$ for the overall HCS but included 0.05 SD in the single site

242 analysis of Massachusetts. For Massachusetts, the number of acceptable allocations

243 corresponding to each constraint is as follows: 644 (.2 SD), 190 (.1 SD), and 44 (.05 SD), out of

24412,870 possible allocations in the unconstrained sampling space.

$246 \quad 2.4 .2$ Data Generation

247 To evaluate Type I error and power, we simulated the number of opioid overdose deaths 248 occurring in the 12-month parallel-arm period first using the following steps:

249 (1) We first obtained the observed baseline number of opioid overdose deaths by averaging 250 number of deaths for the two years prior to the start of the study (2016 and 2017).

251 (2) We then fitted a negative binomial regression model with the observed baseline number of 252 opioid deaths from step (1) as the dependent variable, rural/urban status and state indicators 253 as covariates, and the natural logarithm of population size for each community as the offset. 254 After running the regression, we obtained the fitted value of the intercept $\beta_{0}$, the coefficient 255 for rural/urban status $\beta_{\text {urban }}$, coefficients for state indicators $\beta_{N Y}, \beta_{K Y}, \beta_{M A}$, and the 256 dispersion parameter $\mathrm{k}$.

257 (3) We simulated our outcome variable $Y_{i}$ from a negative binomial distribution with mean

(4) We used the random number generator in R v3.6.2 (R Development Core Team, Vienna, Austria) to simulate 67 values from this distribution, one for each HCS community. 
263 For analyses of the overall HCS, there are 67 communities across 4 states, resulting in a large

264 number of possible allocations for permutation tests. To reduce computational burden, within

265 each of the 5,000 iterations of the simulation, we randomly selected 3000 allocations from the

266 constrained randomization space to estimate the sampling distribution for the permutation

267 statistic.

269 For the subgroup analysis of Massachusetts, we simulated 16 values (one for each MA

270 community) from the negative binomial distribution using the method above without state

271 indicators. Because the actual observed HCS allocation in HCS satisfies the two constraints of

$272 \quad$ 0.2 SD and 0.1 SD, we used the observed HCS allocation as the basis for testing for both of these

273 constraints in order to control the effect of random choice of allocation from the constrained

274 space. For the tighter constraint (0.05 SD), which the observed MA allocation did not satisfy, we

275 randomly selected an allocation from the constrained space. For the single site analysis of

276 Massachusetts, we estimated the sampling distribution for the permutation statistic using the all

277 possible allocations from the constrained space.

279 3. Results

280 In the following, we present the Type I error rate (Table 1) and power (Table 2) for the two 281 model-based tests and the permutation test for the overall HCS and the single-site analysis of

282 Massachusetts subgroup, based on the CCR constraints used by the HCS (i.e. 0.2 SD difference

283 for the continuous covariates population size and baseline opioid death rate).

284 Table 1. Type I error rate for the Model-Based and Permutation Tests for the HEALing 285 Communities Study Design, Overall (4 States) and for the subgroup analysis of Massachusetts 286

\section{Type I error}


${ }^{1}$ Small-sample corrected empirical standard error estimates

\begin{tabular}{|c|c|c|}
\hline \multicolumn{1}{|c|}{ Test Type } & Overall HCS & MA only \\
\hline Model-Based Tests & \multicolumn{2}{|l|}{} \\
\hline Wald-type t-test ${ }^{1}$ & 0.050 & 0.041 \\
\hline Wald-type z-test $^{2}$ & 0.072 & 0.111 \\
\hline Permutation Test & \multicolumn{2}{|l}{} \\
\hline $\begin{array}{l}\text { Difference in } \\
\text { Residuals }\end{array}$ & 0.056 & 0.050 \\
\hline
\end{tabular}

${ }^{2}$ Model-based standard error estimates

Table 2. Power for the Model-Based and Permutation Tests to Detect Various Differences

294 Between Groups in Number of Opioid Overdose Deaths

\begin{tabular}{|c|c|c|c|c|c|c|}
\hline & \multicolumn{6}{|c|}{ Power } \\
\hline & \multicolumn{2}{|c|}{$20 \%$ Difference } & \multicolumn{2}{|c|}{ 30\% Difference } & \multicolumn{2}{|c|}{ 40\% Difference } \\
\hline Test Type & $\begin{array}{c}\text { Overall } \\
\text { HCS }\end{array}$ & MA only & $\begin{array}{c}\text { Overall } \\
\text { HCS }\end{array}$ & MA only & $\begin{array}{c}\text { Overall } \\
\text { HCS }\end{array}$ & $\begin{array}{l}\text { MA } \\
\text { only }\end{array}$ \\
\hline \multicolumn{7}{|l|}{ Model-Based Tests } \\
\hline Wald-type t-test ${ }^{1}$ & 0.792 & 0.397 & 0.989 & 0.774 & 1.000 & 0.880 \\
\hline Wald-type z-test ${ }^{2}$ & 0.822 & 0.595 & 0.995 & 0.902 & 1.000 & 0.960 \\
\hline \multicolumn{7}{|l|}{ Permutation Test } \\
\hline $\begin{array}{l}\text { Difference in } \\
\text { Residuals }\end{array}$ & 0.738 & 0.353 & 0.980 & 0.712 & 1.000 & 0.847 \\
\hline
\end{tabular}

\footnotetext{
${ }^{1}$ Small-sample corrected empirical standard error estimates

${ }^{2}$ Model-based standard error estimates

Note: The constraint used in the CCR is $0.2 \mathrm{SD}$ for population size and baseline opioid death rate.
}

Both the Wald-type t-test using small-sample corrected empirical standard error estimates and the permutation test based on difference in residuals appear to preserve the Type I error for both overall HCS and MA. However, the Wald-type t-test had higher power than the permutation test. The Wald-type z-test with model-based standard error estimates generally had the highest power, however it appeared anti-conservative with Type I errors above 0.05 for both the overall HCS and MA subgroup analysis. Power for all three tests was high (i.e. >0.80), to detect 

differences of at least $30 \%$ between randomized groups in the overall HCS study and to detect a

$30740 \%$ difference for the MA analyses.

309 We also examined the impact of tighter constraints for covariate imbalance on both Type I error

310 (Table 3) and power (Table 4). For the overall HCS, we evaluated a 0.1 SD difference in

311 population size and opioid death rate and for the MA analysis we evaluated constraints of 0.1 SD

312 and 0.05 SD for both covariates. As before, Wald-type t-tests with small-sample corrected

313 empirical standard error estimates preserved the Type I error rates while the Wald-type z-tests

314 with model-based standard error estimates were anti-conservative in terms of Type I error rate.

315 For the permutation tests, Type I error rates appear to be preserved if the constraints used by the

316 CCR are not too tight, otherwise the number of possible allocations in the constrained space may

317 be inadequate. This was observed for the scenario in the MA-only analyses with degree of

318 imbalance set at $0.05 \mathrm{SD}$, resulting in only 44 possible allocations. For a given effect size, the

319 estimated power for each test did not change substantially with tighter constraints for either the

320 overall HCS or the subgroup analysis of MA. The Wald-type t-tests had higher power than the

321 permutation tests in nearly all scenarios.

323 Table 3. Type I error rates based on Maximum Degree of Covariate Imbalance 324

\begin{tabular}{|c|c|c|c|}
\hline & \multicolumn{3}{|c|}{ Type I error } \\
\hline & $\begin{array}{c}\text { Overall } \\
\text { HCS }\end{array}$ & MA only & MA only \\
\hline Max Imbalance & 0.1SD & 0.1SD & 0.05SD \\
\hline Model-Based Tests $^{\mathbf{3}}$ & \multicolumn{3}{|l|}{} \\
\hline Wald-type t-test $^{2}$ & 0.046 & 0.041 & 0.041 \\
\hline Wald-type z-test $^{3}$ & 0.065 & 0.111 & 0.101 \\
\hline Permutation Test & \multicolumn{3}{|l}{} \\
\hline Difference in & 0.047 & 0.050 & 0.087 \\
\hline
\end{tabular}




\section{Residuals}

$325{ }^{1}$ Max imbalance on the constraints for population size and opioid death rate

$326{ }^{2}$ Small-sample corrected empirical standard error estimates

$327{ }^{3}$ Model-based standard error estimates

Table 4. Power based on Maximum Degree of Covariate Imbalance

\begin{tabular}{|c|c|c|c|c|c|c|c|c|c|}
\hline & \multicolumn{3}{|c|}{$20 \%$ Difference } & \multicolumn{3}{|c|}{ 30\% Difference } & \multicolumn{3}{|c|}{ 40\% Difference } \\
\hline & $\begin{array}{c}\text { Overall } \\
\text { HCS }\end{array}$ & $\begin{array}{l}\text { MA } \\
\text { only }\end{array}$ & $\begin{array}{l}\text { MA } \\
\text { only }\end{array}$ & $\begin{array}{c}\text { Overall } \\
\text { HCS }\end{array}$ & $\begin{array}{l}\text { MA } \\
\text { only }\end{array}$ & $\begin{array}{l}\text { MA } \\
\text { only }\end{array}$ & $\begin{array}{c}\text { Overall } \\
\text { HCS }\end{array}$ & $\begin{array}{l}\text { MA } \\
\text { only }\end{array}$ & $\begin{array}{l}\text { MA } \\
\text { only }\end{array}$ \\
\hline Max Imbalance ${ }^{1}$ & 0.1SD & 0.1SD & 0.05SD & 0.1SD & 0.1SD & 0.05SD & 0.1SD & 0.1SD & 0.05SD \\
\hline \multicolumn{10}{|l|}{ Model-Based Tests } \\
\hline Wald-type t-test ${ }^{2}$ & 0.706 & 0.397 & 0.447 & 0.973 & 0.774 & 0.720 & 0.999 & 0.880 & 0.874 \\
\hline Wald-type z-test ${ }^{3}$ & 0.754 & 0.595 & 0.606 & 0.981 & 0.902 & 0.908 & 0.999 & 0.960 & 0.952 \\
\hline \multicolumn{10}{|l|}{ Permutation Test } \\
\hline $\begin{array}{l}\text { Difference in } \\
\text { Residuals }\end{array}$ & 0.657 & 0.353 & 0.335 & 0.941 & 0.712 & 0.721 & 0.996 & 0.847 & 0.813 \\
\hline
\end{tabular}

${ }^{1}$ Max imbalance on the constraints for population size and opioid death rate

${ }^{2}$ Small-sample corrected empirical standard error estimates

${ }^{3}$ Model-based standard error estimates

4. Discussion

338 We examined the performance of model-based and permutation tests in terms of Type I error rate

339 and power under covariate constrained randomization for the HEALing Communities Study

340 (HCS). The HCS is a multi-site parallel group cluster randomized wait-list comparison trial of

341 the Communities That Heal $(\mathrm{CTH})$ intervention evaluating aggregate count outcomes at the

342 community-level (i.e. number of opioid overdose deaths). We assessed the performance of these

343 tests for the primary analyses of the multi-site HCS as well as for a subgroup analysis of a single

344 site, Massachusetts. Additionally, we explored the impact of implementing tighter covariate

345 constraints for the CCR. We found that for both the overall HCS as well as the subgroup of MA,

346 the primary analytic approach using Wald-type t-tests with small-sample corrected empirical 
347 standard error estimates from a negative binomial regression model maintained the proper Type I

348 error rate. The Wald-type z-tests with model-based standard error estimates, however, were anti-

349 conservative. The permutation test based on the difference of average residuals preserved Type I

350 error rates when the degree of constraints was not too tight. Power was high for all tests to detect

351 the hypothesized intervention effect of a $40 \%$ reductions in opioid deaths, both for the overall

352 HCS and for the subgroup analysis of Massachusetts.

354 The key feature of the HCS study that differs from those included in previous research

355 evaluating the performance of statistical tests in cluster randomized trials using CCR is the focus

356 on an aggregate count outcome analyzed using a negative binomial regression model. Li et al.

357 (2016) compared F tests and permutation tests under CCR using linear mixed models [12] and Li

358 et al. (2017) compared four model-based tests and two permutation tests[13] with binary

359 outcomes. Consistent with both of these previous studies, we found that corrected model-based

360 and permutation tests maintain the correct Type I error rate and have similar power when the

361 available allocations in the constrained randomization space is not too small. In the previous

362 research by Li et al. noted above, F tests with linear mixed model, and linearization F-test and

363 KC-corrected GEE t-test with binary outcomes preserved the Type I error rates. In our analysis,

364 the Wald-type t-tests with small-sample corrected empirical standard error estimates preserved

365 the Type I error rates but Wald-type z-tests with model-based standard error estimates did not,

366 emphasizing the importance of the small-sample corrected empirical estimators when using

367 model-based tests in cluster randomized trials with a small number of clusters. The correction

368 factor we used was proposed by Ford and Westgate[19]. They found that by using the average of

369 small-sample corrected empirical estimators proposed by Mancl and DeRouen, and Kauermann 
370 and Carroll [17-20], nominal Type I error rates could be consistently maintained, as confirmed

371 by our simulation study. For permutation tests, Li et al. (2016), and Carter and Hood show that if

372 the constrained randomization space contains larger than 100 possible allocations, permutation

373 tests could maintain the desired Type I errors and high power. Our findings show similar results

374 in that when the constrained space contained $>100$ acceptable allocations, the Type I error rates

375 were preserved and sufficient power was achieved for the hypothesized $30 \%$ and $40 \%$ reductions

376 in opioid deaths for the overall HCS, and for a $40 \%$ reduction in opioid deaths in the subgroup

377 analysis of Massachusetts.

379 Our work has some limitations. First, our goal was to investigate the performance of permutation

380 and model-based tests in the setting of HCS and the results may not be generalizable to other

381 settings as we used community-level outcome data and HCS-specific baseline covariates. In

382 addition, we used an empirical strategy to choose values for the impact of covariates, but did not

383 examine the implications of varying the strength of those covariate relationships.

385 5. Conclusions

386 Based on the results of our simulation study, the Wald-type t-test with small-sample corrected 387 empirical standard error estimates from the negative binomial regression model is a valid and 388 appropriate analytic approach for the HEALing Communities Study. It preserved the Type I error 389 rate and maintained high power for the hypothesized $40 \%$ reduction in opioid deaths for the 390 overall multi-site analyses as well as for a subgroup analysis of a single site. In contrast, Wald391 type z-tests with model-based standard error estimates resulted in inflated Type I error rates. The 392 permutation test based on the difference of average residuals appeared to maintain Type I error 
393 in this setting. However, it had lower power compared to the Wald-type t-test with small-sample

394 corrected empirical standard error estimates along with the added disadvantage of being

395 computationally more complex.

397 6. List of abbreviations

\begin{tabular}{|c|c|}
\hline Full name & Abbreviations \\
\hline Helping to End Addiction Long-term $^{\text {SM }}$ & HEAL \\
\hline The HEALing Communities Study & HCS \\
\hline Communities That Heal & CTH \\
\hline Covariate constrained randomization & CCR \\
\hline Massachusetts & MA \\
\hline
\end{tabular}

398

399

400

401

402

403

404

405

406

407

408

409

410

411 reasonable request and with permission of RTI International.

\section{Competing interests}

413 The authors declare that they have no competing interests.

\section{$414 \quad$ Funding}


415 This research was supported by the National Institutes of Health and the Substance Abuse and

416 Mental Health Services Administration through the NIH HEAL (Helping to End Addiction

417 Long-term ${ }^{\mathrm{SM}}$ ) Initiative under award numbers UM1DA049394, UM1DA049406,

418 UM1DA049412, UM1DA049415, UM1DA049417 (ClinicalTrials.gov Identifier:

419 NCT04111939).

420 Competing interests

421 Debbie Cheng serves on Data Safety Monitoring Boards for Janssen Research \& Development.

422 No other conflicts to disclose.

423 Authors' contributions

424 XT, TH and DC contributed to the concept and study design. XT contributed to the data analysis.

$425 \mathrm{XT}, \mathrm{TH}, \mathrm{PW}, \mathrm{DF}, \mathrm{SF}, \mathrm{NV}$ and DC contributed to the drafting and revising the manuscript.

\section{Acknowledgements}

427 We wish to acknowledge the participation of the HEALing Communities Study communities, 428 community coalitions, and Community Advisory Boards and state government officials who 429 partnered with us on this study. The content is solely the responsibility of the authors and does 430 not necessarily represent the official views of the National Institutes of Health, the Substance 431 Abuse and Mental Health Services Administration or the NIH HEAL Initiative ${ }^{\text {SM }}$.

8. References

1. Murray, D.M., S.P. Varnell, and J.L. Blitstein, Design and analysis of group-randomized trials: a review of recent methodological developments. Am J Public Health, 2004. 94(3): p. 423-32.

2. Rosenberger, W., Randomization in clinical trials. 2002, Wiley: New York.

3. $\quad$ Senn, S., Testing for baseline balance in clinical trials. Stat Med, 1994. 13(17): p. 171526. 
4. Martin, D.C., et al., The effect of matching on the power of randomized community intervention studies. Stat Med, 1993. 12(3-4): p. 329-38.

5. $\quad$ Kernan, W.N., et al., Stratified randomization for clinical trials. J Clin Epidemiol, 1999. 52(1): p. 19-26.

6. Taves, D.R., Minimization: a new method of assigning patients to treatment and control groups. Clin Pharmacol Ther, 1974. 15(5): p. 443-53.

7. Moulton, L.H., Covariate-based constrained randomization of group-randomized trials. Clin Trials, 2004. 1(3): p. 297-305.

8. Raab, G.M. and I. Butcher, Randomization inference for balanced cluster-randomized trials. Clin Trials, 2005. 2(2): p. 130-40.

9. Ludbrook, J., Advantages of permutation (randomization) tests in clinical and experimental pharmacology and physiology. Clin Exp Pharmacol Physiol, 1994. 21(9): p. 673-86.

10. Fu, D., D.M. Murray, and S. Wong, Comparison study of general linear mixed model and permutation tests in group-randomizwd trials under non-normal error distributions, in The Joint Statistical Meetings. 2009: Washington D.C.

11. Murray, D.M., et al., A comparison of permutation and mixed-model regression methods for the analysis of simulated data in the context of a group-randomized trial. Stat Med, 2006. 25(3): p. 375-88.

12. $\mathrm{Li}, \mathrm{F}$., et al., An evaluation of constrained randomization for the design and analysis of group-randomized trials. Stat Med, 2016. 35(10): p. 1565-79.

13. $\mathrm{Li}, \mathrm{F}$., et al., An evaluation of constrained randomization for the design and analysis of group-randomized trials with binary outcomes. Stat Med, 2017. 36(24): p. 3791-3806.

14. El-Bassel, N., et al., Introduction to the special issue on the HEALing Communities Study. Drug Alcohol Depend, 2020. 217: p. 108327.

15. Chandler, R.K., et al., Addressing opioid overdose deaths: The vision for the HEALing communities study. Drug Alcohol Depend, 2020. 217: p. 108329.

16. Walsh, S.L., N. El-Bassel, and R.D. Jackson, The healing (helping to end addiction longterm sm) communities study: Protocol for a cluster randomized trial at the community level to reduce opioid overdose deaths through implementation of an integrated set of evidence-based practices. Drug and Alcohol Dependence, 2020. 217: p. 108335.

17. Kauermann, G. and R.J. Carroll, A note on the efficiency of sandwich covariance matrix estimation. Journal of the American Statistical Association, 2001. 96: p. 1387-1396.

18. Mancl, L.A. and T.A. DeRouen, A covariance estimator for GEE with improved smallsample properties. Biometrics, 2001. 57(1): p. 126-34.

19. Ford, W.P. and P.M. Westgate, Improved standard error estimator for maintaining the validity of inference in cluster randomized trials with a small number of clusters. Biom J, 2017. 59(3): p. 478-495.

20. Fay, M.P. and B.I. Graubard, Small-sample adjustments for Wald-type tests using sandwich estimators. Biometrics, 2001. 57(4): p. 1198-206.

21. Good, P.I., Parametric and Bootstrap Tests of Hypotheses. 2005: Springer.

22. Gail, M.H., et al., On design considerations and randomization-based inference for community intervention trials. Stat Med, 1996. 15(11): p. 1069-92. 